\title{
SOME NEW DOUBLE SEQUENCE SPACES OF INVARIANT MEANS
}

\author{
M. Mursaleen and S.A. Mohiuddine \\ Aligarh Muslim University, India
}

\begin{abstract}
In this paper, we define some new spaces of double sequences involving the idea of $\sigma$-mean; i.e., we define the spaces of strongly $\sigma$-convergent, absolutely $\sigma$-almost convergent, $\sigma$-almost bounded and absolutely $\sigma$-convergent double sequences and establish some inclusion relations along with some numerical examples.
\end{abstract}

\section{INTRODUCTION AND PRELIMINARIES}

First, we recall some notations and definitions which will be used throughout the paper (cf. [12]).

A double sequence $x=\left(x_{j k}\right)$ of real or complex numbers is said to be bounded if $\|x\|_{\infty}=\sup _{j, k}\left|x_{j k}\right|<\infty$. We denote the space of all bounded double sequences by $\mathcal{L}^{\infty}$.

A double sequence $x=\left(x_{j k}\right)$ is said to converge to the limit $L$ in Pringsheim's sense (shortly, p-convergent to $L$ ) if for every $\varepsilon>0$ there exists an integer $N$ such that $\left|x_{j k}-L\right|<\varepsilon$ whenever $j, k>N$. In this case $L$ is called the $p$-limit of $x$. If in addition $x \in \mathcal{L}^{\infty}$, then $x$ is said to be boundedly p-convergent to $L$ in Pringsheim's sense (shortly, bp-convergent to $L$ ).

In general, for any notion of convergence $\nu$, the space of all $\nu$-convergent double sequences will be denoted by $\mathcal{C}_{\nu}$ and the limit of a $\nu$-convergent double sequence $x$ by $\nu$-lim $x_{j k}$, where $\nu \in\{p, b p\}$.

Let $l_{\infty}$ and $c$ be the spaces of bounded and convergent sequences $x=\left(x_{k}\right)$ respectively. Let $\sigma$ be a one-to-one mapping from the set $\mathbb{N}$ of natural numbers

2010 Mathematics Subject Classification. 40C05, 40H05.

Key words and phrases. Double sequences, absolute almost convergence, $\sigma$-mean, strong $\sigma$-convergence, absolute $\sigma$-convergence, $\sigma$-almost boundedness, absolute $\sigma$-convergence. 
into itself. A continuous linear functional $\phi$ on the space $l_{\infty}$ is said to be an invariant mean or a $\sigma$-mean if and only if $(i) \phi(x) \geq 0$ when the sequence $x=\left(x_{k}\right)$ has $x_{k} \geq 0$ for all $k,(i i) \phi(e)=1$, where $e=(1,1,1, \cdots)$, and (iii) $\phi(x)=\phi\left(\left(x_{\sigma(k)}\right)\right)$ for all $x \in l_{\infty}$. Throughout this paper we consider the mapping $\sigma$ which has no finite orbits, that is, $\sigma^{p}(k) \neq k$ for all integer $k \geq 0$ and $p \geq 1$, where $\sigma^{p}(k)$ denotes the $p t h$ iterate of $\sigma$ at $k$. Note that, a $\sigma$-mean extends the limit functional on the space $c$ in the sense that $\phi(x)=\lim x$ for all $x \in c$, (cf. [10]). Consequently, $c \subset V_{\sigma}$ the set of bounded sequences all of whose $\sigma$-means are equal.

The idea of $\sigma$-convergence for double sequences has recently been introduced in [3] and further studied by Mursaleen and Mohiuddine ([8,9]). A double sequence $x=\left(x_{j k}\right)$ of real numbers is said to be $\sigma$-convergent to a number $L$ if and only if $\sigma$-lim $x=b p$ - $\lim _{p, q \rightarrow \infty} d_{p q s t}(x)=L$ uniformly in $s, t$, where

$$
\begin{aligned}
d_{p q s t}(x) & =\frac{1}{(p+1)(q+1)} \sum_{j=0}^{p} \sum_{k=0}^{q} x_{\sigma^{j}(s), \sigma^{k}(t)}, s, t=0,1,2, \ldots ; \\
d_{0,0, s, t}(x) & =x_{s t}, d_{-1,0, s, t}(x)=x_{s-1, t}, d_{0,-1, s, t}(x) \\
& =x_{s, t-1}, d_{-1,-1, s, t}(x)=x_{s-1, t-1},
\end{aligned}
$$

and $x_{\sigma^{j}(s), \sigma^{k}(t)}=0$ for all $j$ or $k$ or both negative.

Let us denote by $\mathcal{V}_{\sigma}$ the space of $\sigma$-convergent double sequences $x=\left(x_{j k}\right)$, i.e.,

$\mathcal{V}_{\sigma}=\left\{x \in \mathcal{L}^{\infty}: b p\right.$ - $\lim _{p, q \rightarrow \infty} d_{p q s t}(x)=L$ uniformly in $\left.s, t ; L=\sigma-\lim x\right\}$.

For $\sigma(n)=n+1$, the set $\mathcal{V}_{\sigma}$ is reduced to the set $f_{2}$ of almost convergent double sequences ([7]). Note that $\mathcal{C}_{b p} \subset \mathcal{V}_{\sigma} \subset \mathcal{L}^{\infty}$.

In this paper we define the strong $\sigma$-convergence for double sequences which is an extension of the idea of strong $\sigma$-convergence for single sequences due to Mursaleen ([11]). We also introduce some more new spaces of double sequences; e.g., absolute $\sigma$-almost convergence, $\sigma$-almost boundedness and absolute $\sigma$-convergence involving the idea of invariant mean and we find relations among these spaces. We also construct some examples of double sequences to support our claims.

\section{Some NEW SEQUENCE SPACES}

In this section, we define some double sequence spaces. Some of such spaces for single sequences have been studied in $[4,5,10,11]$.

For any given infinite double series $\sum_{s} \sum_{t} a_{s t}$, denoted as " $a$ ", we write

$$
x_{j k}=\sum_{s=1}^{j} \sum_{t=1}^{k} a_{s t}, j, k=1,2, \ldots,
$$


and

$$
\begin{aligned}
& \phi_{p q s t}(x)=d_{p q s t}(x)-d_{p-1, q, s, t}(x)-d_{p, q-1, s, t}(x)+d_{p-1, q-1, s, t}(x) \\
& =\frac{1}{(p+1)(q+1)} \sum_{j=0}^{p} \sum_{k=0}^{q} x_{\sigma^{j}(s), \sigma^{k}(t)}-\frac{1}{p(q+1)} \sum_{j=0}^{p-1} \sum_{k=0}^{q} x_{\sigma^{j}(s), \sigma^{k}(t)} \\
& -\frac{1}{(p+1) q} \sum_{j=0}^{p} \sum_{k=0}^{q-1} x_{\sigma^{j}(s), \sigma^{k}(t)}+\frac{1}{p q} \sum_{j=0}^{p-1} \sum_{k=0}^{q-1} x_{\sigma^{j}(s), \sigma^{k}(t)} \\
& =\frac{1}{(q+1)} \sum_{k=0}^{q}\left[\frac{1}{(p+1)} \sum_{j=0}^{p} x_{\sigma^{j}(s), \sigma^{k}(t)}-\frac{1}{p} \sum_{j=0}^{p-1} x_{\sigma^{j}(s), \sigma^{k}(t)}\right] \\
& -\frac{1}{q} \sum_{k=0}^{q-1}\left[\frac{1}{(p+1)} \sum_{j=0}^{p} x_{\sigma^{j}(s), \sigma^{k}(t)}-\frac{1}{p} \sum_{j=0}^{p-1} x_{\sigma^{j}(s), \sigma^{k}(t)}\right] \\
& =\frac{1}{(q+1)} \sum_{k=0}^{q}\left[\frac{1}{p(p+1)} \sum_{j=1}^{p} j\left(x_{\sigma^{j}(s), \sigma^{k}(t)}-x_{\sigma^{j-1}(s), \sigma^{k}(t)}\right)\right] \\
& -\frac{1}{q} \sum_{k=0}^{q-1}\left[\frac{1}{p(p+1)} \sum_{j=1}^{p} j\left(x_{\sigma^{j}(s), \sigma^{k}(t)}-x_{\sigma^{j-1}(s), \sigma^{k}(t)}\right)\right] \\
& =\frac{1}{p(p+1)} \sum_{j=1}^{p} j\left[\frac{1}{(q+1)} \sum_{k=0}^{q}-\frac{1}{q} \sum_{k=0}^{q-1}\right]\left(x_{\sigma^{j}(s), \sigma^{k}(t)}-x_{\sigma^{j-1}(s), \sigma^{k}(t)}\right) \\
& =\frac{1}{p(p+1)} \sum_{j=1}^{p} j\left[\frac{1}{(q+1)} \sum_{k=0}^{q} y_{\sigma^{j}(s), \sigma^{k}(t)}-\frac{1}{q} \sum_{k=0}^{q-1} y_{\sigma^{j}(s), \sigma^{k}(t)}\right] \text {, }
\end{aligned}
$$

where $y_{\sigma^{j}(s), \sigma^{k}(t)}=\left(x_{\sigma^{j}(s), \sigma^{k}(t)}-x_{\sigma^{j-1}(s), \sigma^{k}(t)}\right)$, solving further as above, we get

$$
\begin{aligned}
\phi_{p q s t}(x)= & \frac{1}{p(p+1)} \sum_{j=1}^{p} j\left[\frac{1}{q(q+1)} \sum_{k=1}^{q} k\left(y_{\sigma^{j}(s), \sigma^{k}(t)}-y_{\sigma^{j}(s), \sigma^{k-1}(t)}\right)\right] \\
= & \frac{1}{p(p+1) q(q+1)} \sum_{j=1}^{p} \sum_{k=1}^{q} j k\left[x_{\sigma^{j}(s), \sigma^{k}(t)}-x_{\sigma^{j-1}(s), \sigma^{k}(t)}\right. \\
& \left.-x_{\sigma^{j}(s), \sigma^{k-1}(t)}+x_{\sigma^{j-1}(s), \sigma^{k-1}(t)}\right] .
\end{aligned}
$$


Now we define

$$
\phi_{p q s t}(x)=\left\{\begin{array}{l}
\frac{1}{p(p+1) q(q+1)} \sum_{j=1}^{p} \sum_{k=1}^{q} j k\left[x_{\sigma^{j}(s), \sigma^{k}(t)}-x_{\sigma^{j-1}(s), \sigma^{k}(t)}\right. \\
\left.-x_{\sigma^{j}(s), \sigma^{k-1}(t)}+x_{\sigma^{j-1}(s), \sigma^{k-1}(t)}\right] ; \quad p, q \geq 1, \\
a_{s t} ; \quad p \text { or } q \text { or both zero. }
\end{array}\right.
$$

Throughout we take $x_{s t}=0$ if either $s$ or $t$ or both are zero or negative.

Note that throughout the present work the 'limit' means 'bp-limit'.

Definition 2.1. A bounded double sequence $x=\left(x_{j k}\right)$ is said to be strongly $\sigma$-convergent if there exists a number $\ell$ such that

$$
\frac{1}{(p+1)(q+1)} \sum_{j=0}^{p} \sum_{k=0}^{q}\left|x_{\sigma^{j}(s), \sigma^{k}(t)}-\ell\right| \longrightarrow 0 \text { as } p, q \longrightarrow \infty \text { uniformly in } s, t .
$$

In this case, we write $\left[\mathcal{V}_{\sigma}\right]-\lim x=\ell$. Let us denote by $\left[\mathcal{V}_{\sigma}\right]$ the set of all strongly $\sigma$-convergent sequences $x=\left(x_{j k}\right)$.

REMARK 2.2. If $\left[\mathcal{V}_{\sigma}\right]-\lim x=\ell$, that is

$$
\frac{1}{(p+1)(q+1)} \sum_{j=0}^{p} \sum_{k=0}^{q}\left|x_{\sigma^{j}(s), \sigma^{k}(t)}-\ell\right| \longrightarrow 0
$$

as $p, q \longrightarrow \infty$, uniformly in $s, t$; then

$$
\frac{1}{(p+1)(q+1)} \sum_{j=0}^{p} \sum_{k=0}^{q}\left|\frac{1}{k+1} \sum_{v=0}^{k} x_{\sigma^{j}(s), \sigma^{v}(t)}-\ell\right| \longrightarrow 0
$$

and

$$
\frac{1}{(p+1)(q+1)} \sum_{j=0}^{p} \sum_{k=0}^{q}\left|\frac{1}{j+1} \sum_{u=0}^{j} x_{\sigma^{u}(s), \sigma^{k}(t)}-\ell\right| \longrightarrow 0
$$

as $p, q \longrightarrow \infty$, uniformly in $s, t$.

REMARK 2.3. For $\sigma(n)=n+1$, the set $\left[\mathcal{V}_{\sigma}\right]$ is reduced to the set $\left[f_{2}\right]$ of strong almost convergent double sequences $([2])$. Note that

(a) $\mathcal{C}_{b p} \subset\left[\mathcal{V}_{\sigma}\right]$ and $b p-\lim x=\left[\mathcal{V}_{\sigma}\right]-\lim x$;

(b) $\left[\mathcal{V}_{\sigma}\right] \subset \mathcal{V}_{\sigma}$ and $\sigma-\lim x=\left[\mathcal{V}_{\sigma}\right]-\lim x$

(c) $\left[\mathcal{V}_{\sigma}\right]$-limit is unique.

Definition 2.4. A bounded double sequence $x=\left(x_{j k}\right)$ or the series a is said to be absolutely $\sigma$-almost convergent if

$$
\sum_{p=1}^{\infty} \sum_{q=1}^{\infty}\left|\phi_{p q s t}(x)\right| \text { converges uniformly in } s, t .
$$


By $\mathcal{W}_{\sigma}$, we denote the set of all absolutely $\sigma$-almost convergent double sequences. For $\sigma(n)=n+1$, we obtain the space of absolutely almost convergent double sequences. The concept of absolutely almost convergence for single sequences was introduced by Das, Kuttner and Nanda ([4]). Note that $\mathcal{W}_{\sigma} \subset \mathcal{V}_{\sigma}$.

Definition 2.5. A bounded double sequence $x=\left(x_{j k}\right)$ is said to be $\sigma$ almost bounded if

$$
\sup _{s, t} \sum_{p=0}^{\infty} \sum_{q=0}^{\infty}\left|\phi_{p q s t}(x)\right|<\infty
$$

By $\mathcal{U}_{\sigma}$, we denote the set of all $\sigma$-almost bounded double sequences.

Definition 2.6. A bounded double series a (i.e., $\sum_{s} \sum_{t} a_{s t}$ ) is said to be absolutely $\sigma$-convergent if

$$
\sum_{j=0}^{\infty} \sum_{k=0}^{\infty}\left|x_{\sigma^{j}(s), \sigma^{k}(t)}-x_{\sigma^{j-1}(s), \sigma^{k}(t)}-x_{\sigma^{j}(s), \sigma^{k-1}(t)}+x_{\sigma^{j-1}(s), \sigma^{k-1}(t)}\right|<\infty,
$$

uniformly in $s, t$, where $\left(x_{j k}\right)$ is a double sequence of partial sums of the series $\sum_{s} \sum_{t} a_{s t}$, i.e., $x_{j k}=\sum_{s=1}^{j} \sum_{t=1}^{k} a_{s t}$; and $x_{\sigma^{j-1}(s), \sigma^{k-1}(t)}=0$ for $j=0$ or $/$ and $k=0$. By $\mathcal{L}_{\sigma}$, we denote the set of all absolutely $\sigma$-convergent double series. For $\sigma(n)=n+1$, it reduces to the set of absolutely almost convergent double series.

\section{LEMMAS}

To prove our main results, we need first to prove some lemmas.

LEMMA 3.1 (Abel's transformation for double summation).

$$
\begin{gathered}
\sum_{j=1}^{p} \sum_{k=1}^{q} v_{j k}\left(u_{j k}-u_{j+1, k}-u_{j, k+1}+u_{j+1, k+1}\right) \\
=\sum_{j=1}^{p} \sum_{k=1}^{q} u_{j k}\left(\triangle_{11} v_{j k}\right)-\sum_{j=1}^{p} u_{j, q+1}\left(\triangle_{10} v_{j q}\right) \\
\quad-\sum_{k=1}^{q} u_{p+1, k}\left(\triangle_{01} v_{p k}\right)+u_{p+1, q+1} v_{p q}
\end{gathered}
$$

where

$$
\triangle_{10} v_{j q}=v_{j q}-v_{j-1, q}, \triangle_{01} v_{p k}=v_{p k}-v_{p, k-1}
$$

and

$$
\triangle_{11} v_{j k}=v_{j k}-v_{j-1, k}-v_{j, k-1}+v_{j-1, k-1} .
$$


Proof. Abel's transformation for single summation is

$$
\sum_{i=1}^{m} v_{i}\left(u_{i} \mp u_{i+1}\right)=\sum_{i=1}^{m} u_{i}\left(v_{i} \mp v_{i-1}\right) \mp u_{m+1} v_{m} .
$$

Now we find Abel's transformation for double summation,

$$
\begin{aligned}
& \sum_{j=1}^{p} \sum_{k=1}^{q} v_{j k}\left(u_{j k}-u_{j+1, k}-u_{j, k+1}+u_{j+1, k+1}\right) \\
& \quad=\sum_{k=1}^{q}\left[\sum_{j=1}^{p} v_{j k}\left(u_{j k}-u_{j+1, k}\right)-\sum_{j=1}^{p} v_{j k}\left(u_{j, k+1}-u_{j+1, k+1}\right)\right] .
\end{aligned}
$$

By using (3.1), we have

$$
\begin{aligned}
\sum_{j=1}^{p} & \sum_{k=1}^{q} v_{j k}\left(u_{j k}-u_{j+1, k}-u_{j, k+1}+u_{j+1, k+1}\right) \\
= & \sum_{k=1}^{q}\left[\sum_{j=1}^{p} u_{j k}\left(v_{j k}-v_{j-1, k}\right)-u_{p+1, k} v_{p k}\right. \\
& \left.-\sum_{j=1}^{p} u_{j, k+1}\left(v_{j k}-v_{j-1, k}\right)+u_{p+1, k+1} v_{p k}\right] \\
= & \sum_{j=1}^{p}\left[\sum_{k=1}^{q} v_{j k}\left(u_{j k}-u_{j, k+1}\right)-\sum_{k=1}^{q} v_{j-1, k}\left(u_{j k}-u_{j, k+1}\right)\right] \\
& -\sum_{k=1}^{q} u_{p+1, k} v_{p k}+\sum_{k=1}^{q} u_{p+1, k+1} v_{p k},
\end{aligned}
$$

and now again using (3.1), we get

$$
\begin{aligned}
\sum_{j=1}^{p} & \sum_{k=1}^{q} v_{j k}\left(u_{j k}-u_{j+1, k}-u_{j, k+1}+u_{j+1, k+1}\right) \\
= & \sum_{j=1}^{p}\left[\sum_{k=1}^{q} u_{j k}\left(v_{j k}-v_{j, k-1}\right)-u_{j, q+1} v_{j q}\right. \\
& \left.-\sum_{k=1}^{q} u_{j k}\left(v_{j-1, k}-v_{j-1, k-1}\right)+u_{j, q+1} v_{j-1, q}\right] \\
& -\sum_{k=1}^{q} u_{p+1, k} v_{p k}+\sum_{k=1}^{q} u_{p+1, k} v_{p, k-1}+u_{p+1, q+1} v_{p q}
\end{aligned}
$$




$$
\begin{aligned}
= & \sum_{j=1}^{p} \sum_{k=1}^{q} u_{j k}\left(v_{j k}-v_{j, k-1}-v_{j-1, k}+v_{j-1, k-1}\right)-\sum_{j=1}^{p} u_{j, q+1} v_{j q} \\
& +\sum_{j=1}^{p} u_{j, q+1} v_{j-1, q}-\sum_{k=1}^{q} u_{p+1, k} v_{p k}+\sum_{k=1}^{q} u_{p+1, k} v_{p, k-1}+u_{p+1, q+1} v_{p q} \\
= & \sum_{j=1}^{p} \sum_{k=1}^{q} u_{j k}\left(\triangle_{11} v_{j k}\right)-\sum_{j=1}^{p} u_{j, q+1}\left(\triangle_{10} v_{j q}\right) \\
& -\sum_{k=1}^{q} u_{p+1, k}\left(\triangle_{01} v_{p k}\right)+u_{p+1, q+1} v_{p q} .
\end{aligned}
$$

This completes the proof of the lemma.

Another form of Abel's transformation for double summation is given by Altay and Başar ([1]) and by Hardy ([6]).

LEMMA 3.2. $\left[\mathcal{V}_{\sigma}\right]-\lim x=\ell$ if and only if

(i) $\sigma-\lim x=\ell$;

(ii) $\frac{1}{p q} \sum_{j=1}^{p} \sum_{k=1}^{q}|\alpha(j, k, s, t)-\ell| \longrightarrow 0(p, q \longrightarrow \infty)$ uniformly in $s, t$;

(iii) $\frac{1}{p q} \sum_{j=1}^{p} \sum_{k=1}^{q}|\beta(j, k, s, t)-\ell| \longrightarrow 0(p, q \longrightarrow \infty)$ uniformly in $s, t$;

(iv) $\frac{1}{p q} \sum_{j=1}^{p} \sum_{k=1}^{q}\left|x_{\sigma^{j}(s), \sigma^{k}(t)}+d_{j k s t}-\alpha(j, k, s, t)-\beta(j, k, s, t)\right| \longrightarrow 0(p, q \longrightarrow$ $\infty)$

uniformly in $s, t$, where

$\alpha(j, k, s, t)=\frac{1}{(j+1)} \sum_{u=0}^{j} x_{\sigma^{u}(s), \sigma^{k}(t)}$ and $\beta(j, k, s, t)=\frac{1}{(k+1)} \sum_{v=0}^{k} x_{\sigma^{j}(s), \sigma^{v}(t)}$.

Proof. Let $\left[\mathcal{V}_{\sigma}\right]-\lim x=\ell$. Then obviously $\sigma$-lim $x=\ell$ by Remark 2.3(b). From the Remark 2.2, (ii) and (iii) follow immediately. Now

$$
\begin{aligned}
& \frac{1}{p q} \sum_{j=1}^{p} \sum_{k=1}^{q}\left|x_{\sigma^{j}(s), \sigma^{k}(t)}+d_{j k s t}(x)-\alpha(j, k, s, t)-\beta(j, k, s, t)\right| \\
& =\frac{1}{p q} \sum_{j=1}^{p} \sum_{k=1}^{q}\left|x_{\sigma^{j}(s), \sigma^{k}(t)}-\ell+d_{j k s t}(x)-\ell-\alpha(j, k, s, t)+\ell-\beta(j, k, s, t)+\ell\right| \\
& \leq \frac{1}{p q} \sum_{j=1}^{p} \sum_{k=1}^{q}\left(\left|x_{\sigma^{j}(s), \sigma^{k}(t)}-\ell\right|+\left|d_{j k s t}(x)-\ell\right|\right. \\
& \quad+|\alpha(j, k, s, t)-\ell|+|\beta(j, k, s, t)-\ell|) \\
& \quad \longrightarrow 0 \text { as } p, q \longrightarrow \infty, \text { uniformly in } s, t ;
\end{aligned}
$$


since

(a) $\left[\mathcal{V}_{\sigma}\right]$-lim $x=\ell$ imply that the first sum tends to zero;

(b) (ii) and (iii) imply that the third and the fourth sums tend to zero;

(c) (i) implies that $d_{j k s t}(x) \longrightarrow \ell(j, k \longrightarrow \infty)$ uniformly in $s, t$; and so the second sum tends to zero.

Conversely, suppose that the conditions hold. Now

$$
\begin{aligned}
& \frac{1}{p q} \sum_{j=1}^{p} \sum_{k=1}^{q}\left|x_{\sigma^{j}(s), \sigma^{k}(t)}-\ell\right| \\
& \leq \frac{1}{p q} \sum_{j=1}^{p} \sum_{k=1}^{q}\left|x_{\sigma^{j}(s), \sigma^{k}(t)}+d_{j k s t}(x)-\alpha(j, k, s, t)-\beta(j, k, s, t)\right| \\
& \quad+\frac{1}{p q} \sum_{j=1}^{p} \sum_{k=1}^{q}\left|d_{j k s t}(x)-\ell\right|+\frac{1}{p q} \sum_{j=1}^{p} \sum_{k=1}^{q}|\alpha(j, k, s, t)-\ell| \\
& \quad+\frac{1}{p q} \sum_{j=1}^{p} \sum_{k=1}^{q}|\beta(j, k, s, t)-\ell| \\
& \quad \longrightarrow 0 \text { as } p, q \longrightarrow \infty, \text { uniformly in } s, t .
\end{aligned}
$$

This completes the proof of the lemma.

LEMMA 3.3. We have

$$
\begin{aligned}
& x_{\sigma^{j}(s), \sigma^{k}(t)}+d_{j k s t}(x)-\alpha(j, k, s, t)-\beta(j, k, s, t) \\
& \quad=j k\left[d_{j k s t}(x)-d_{j-1, k, s, t}(x)-d_{j, k-1, s, t}(x)+d_{j-1, k-1, s, t}(x)\right] .
\end{aligned}
$$

Proof. We have

$$
\begin{aligned}
& d_{j k s t}(x)-d_{j-1, k, s, t}(x)-d_{j, k-1, s, t}(x)+d_{j-1, k-1, s, t}(x) \\
&= {\left[\frac{1}{(j+1)(k+1)} \sum_{u=0}^{j} \sum_{v=0}^{k} x_{\sigma^{u}(s), \sigma^{v}(t)}-\frac{1}{j(k+1)} \sum_{u=0}^{j-1} \sum_{v=0}^{k} x_{\sigma^{u}(s), \sigma^{v}(t)}\right] } \\
&3.2)-\left[\frac{1}{(j+1) k} \sum_{u=0}^{j} \sum_{v=0}^{k-1} x_{\sigma^{u}(s), \sigma^{v}(t)}-\frac{1}{j k} \sum_{u=0}^{j-1} \sum_{v=0}^{k-1} x_{\sigma^{u}(s), \sigma^{v}(t)}\right] .
\end{aligned}
$$

First we simplify the expression in the first brackets

$$
\begin{aligned}
& {\left[\frac{1}{(j+1)(k+1)} \sum_{u=0}^{j} \sum_{v=0}^{k} x_{\sigma^{u}(s), \sigma^{v}(t)}-\frac{1}{j(k+1)} \sum_{u=0}^{j-1} \sum_{v=0}^{k} x_{\sigma^{u}(s), \sigma^{v}(t)}\right]} \\
& =\frac{1}{j(j+1)(k+1)}\left[\sum_{v=0}^{k}\left(j \sum_{u=0}^{j} x_{\sigma^{u}(s), \sigma^{v}(t)}-(j+1) \sum_{u=0}^{j-1} x_{\sigma^{u}(s), \sigma^{v}(t)}\right)\right]
\end{aligned}
$$




$$
\begin{aligned}
& =\frac{1}{j(j+1)(k+1)} \sum_{v=0}^{k}\left[j x_{\sigma^{j}(s), \sigma^{v}(t)}-\sum_{u=0}^{j-1} x_{\sigma^{u}(s), \sigma^{v}(t)}\right] \\
& =\frac{1}{j(j+1)(k+1)} \sum_{v=0}^{k}\left[(j+1) x_{\sigma^{j}(s), \sigma^{v}(t)}-\sum_{u=0}^{j} x_{\sigma^{u}(s), \sigma^{v}(t)}\right] \\
& =\frac{1}{j(k+1)} \sum_{v=0}^{k}\left[x_{\sigma^{j}(s), \sigma^{v}(t)}-\frac{1}{(j+1)} \sum_{u=0}^{j} x_{\sigma^{u}(s), \sigma^{v}(t)}\right] \\
& =\frac{1}{j(k+1)} \sum_{v=0}^{k} x_{\sigma^{j}(s), \sigma^{v}(t)}-\frac{1}{j(j+1)(k+1)} \sum_{u=0}^{j} \sum_{v=0}^{k} x_{\sigma^{u}(s), \sigma^{v}(t)} \\
& =\frac{1}{j(k+1)} \sum_{v=0}^{k} x_{\sigma^{j}(s), \sigma^{v}(t)}-\frac{1}{j} d_{j k s t}(x) .
\end{aligned}
$$

Similarly the expression in the second brackets can be simplified by replacing $k-1$ for $k$, i.e.,

$$
\begin{gathered}
\frac{1}{(j+1) k} \sum_{u=0}^{j} \sum_{v=0}^{k-1} x_{\sigma^{u}(s), \sigma^{v}(t)}-\frac{1}{j k} \sum_{u=0}^{j-1} \sum_{v=0}^{k-1} x_{\sigma^{u}(s), \sigma^{v}(t)} \\
=\frac{1}{j k} \sum_{v=0}^{k-1} x_{\sigma^{j}(s), \sigma^{v}(t)}-\frac{1}{j} d_{j, k-1, s, t}(x) .
\end{gathered}
$$

Substituting (3.3) and (3.4) in (3.2), we get

$$
\begin{aligned}
d_{j k s t}(x)-d_{j-1, k, s, t}(x)-d_{j, k-1, s, t}(x)+d_{j-1, k-1, s, t}(x) \\
=\frac{1}{j(k+1)} \sum_{v=0}^{k} x_{\sigma^{j}(s), \sigma^{v}(t)}-\frac{1}{j k} \sum_{v=0}^{k-1} x_{\sigma^{j}(s), \sigma^{v}(t)} \\
\quad-\frac{1}{j} d_{j k s t}(x)+\frac{1}{j} d_{j, k-1, s, t}(x) \\
=\frac{1}{j k(k+1)}\left[k \sum_{v=0}^{k} x_{\sigma^{j}(s), \sigma^{v}(t)}-(k+1) \sum_{v=0}^{k-1} x_{\sigma^{j}(s), \sigma^{v}(t)}\right] \\
\quad-\frac{1}{j}\left(d_{j k s t}(x)-d_{j, k-1, s, t}(x)\right) \\
=\frac{1}{j k(k+1)}\left[k x_{\sigma^{j}(s), \sigma^{k}(t)}-\sum_{v=0}^{k-1} x_{\sigma^{j}(s), \sigma^{v}(t)}\right]-\frac{1}{j}\left(d_{j k s t}(x)-d_{j, k-1, s, t}(x)\right)
\end{aligned}
$$




$$
\begin{aligned}
= & \frac{1}{j k(k+1)}\left[(k+1) x_{\sigma^{j}(s), \sigma^{k}(t)}-\sum_{v=0}^{k} x_{\sigma^{j}(s), \sigma^{v}(t)}\right] \\
& -\frac{1}{j}\left(d_{j k s t}(x)-d_{j, k-1, s, t}(x)\right)
\end{aligned}
$$

$$
=\frac{1}{j k}\left[x_{\sigma^{j}(s), \sigma^{k}(t)}-\frac{1}{(k+1)} \sum_{v=0}^{k} x_{\sigma^{j}(s), \sigma^{v}(t)}\right]-\frac{1}{j}\left(d_{j k s t}(x)-d_{j, k-1, s, t}(x)\right) .
$$

We know that

$$
\begin{aligned}
d_{j k s t}(x) & =\frac{1}{(j+1)(k+1)} \sum_{u=0}^{j} \sum_{v=0}^{k} x_{\sigma^{u}(s), \sigma^{v}(t)} \\
& =\frac{1}{(j+1)(k+1)}\left[\sum_{u=0}^{j-1} \sum_{v=0}^{k} x_{\sigma^{u}(s), \sigma^{v}(t)}+\sum_{v=0}^{k} x_{\sigma^{j}(s), \sigma^{v}(t)}\right]
\end{aligned}
$$

and

$$
d_{j-1, k, s, t}(x)=\frac{1}{j(k+1)} \sum_{u=0}^{j-1} \sum_{v=0}^{k} x_{\sigma^{u}(s), \sigma^{v}(t)} .
$$

From (3.6) and (3.7), we have

$$
(j+1) d_{j k s t}(x)-j d_{j-1, k, s, t}(x)=\frac{1}{(k+1)} \sum_{v=0}^{k} x_{\sigma^{j}(s), \sigma^{v}(t)} .
$$

Thus (3.5) becomes

$$
\begin{aligned}
& d_{j k s t}(x)-d_{j-1, k, s, t}(x)-d_{j, k-1, s, t}(x)+d_{j-1, k-1, s, t}(x) \\
&= \frac{1}{j k}\left[x_{\sigma^{j}(s), \sigma^{k}(t)}-(j+1) d_{j k s t}(x)+j d_{j-1, k, s, t}(x)\right] \\
&-\frac{1}{j}\left(d_{j k s t}(x)-d_{j, k-1, s, t}(x)\right) \\
&= \frac{1}{j k}\left[x_{\sigma^{j}(s), \sigma^{k}(t)}-d_{j k s t}(x)-j\left(d_{j k s t}(x)-d_{j-1, k, s, t}(x)\right)\right.
\end{aligned}
$$

Also (3.8) can be written as

$$
j\left(d_{j k s t}(x)-d_{j-1, k, s, t}(x)\right)=\frac{1}{(k+1)} \sum_{v=0}^{k} x_{\sigma^{j}(s), \sigma^{v}(t)}-d_{j k s t}(x) .
$$

Similarly we can write

$$
k\left(d_{j k s t}(x)-d_{j, k-1, s, t}(x)\right)=\frac{1}{(j+1)} \sum_{u=0}^{j} x_{\sigma^{u}(s), \sigma^{k}(t)}-d_{j k s t}(x) .
$$


Using (3.10) and (3.11) in (3.9), we get

$$
\begin{aligned}
d_{j k s t}(x) & -d_{j-1, k, s, t}(x)-d_{j, k-1, s, t}(x)+d_{j-1, k-1, s, t}(x) \\
= & \frac{1}{j k}\left[x_{\sigma^{j}(s), \sigma^{k}(t)}+d_{j k s t}(x)-\frac{1}{(j+1)} \sum_{u=0}^{j} x_{\sigma^{u}(s), \sigma^{k}(t)}\right. \\
& \left.-\frac{1}{(k+1)} \sum_{v=0}^{k} x_{\sigma^{j}(s), \sigma^{v}(t)}\right]
\end{aligned}
$$

which implies that

$$
\begin{aligned}
& x_{\sigma^{j}(s), \sigma^{k}(t)}+d_{j k s t}(x)-\alpha(j, k, s, t)-\beta(j, k, s, t) \\
& \quad=j k\left[d_{j k s t}(x)-d_{j-1, k, s, t}(x)-d_{j, k-1, s, t}(x)+d_{j-1, k-1, s, t}(x)\right] .
\end{aligned}
$$

This completes the proof of the lemma.

LEMMA 3.4. Let

$$
\alpha_{g h s t}=\sum_{j=g}^{\infty} \sum_{k=h}^{\infty}\left|d_{j k s t}(x)-d_{j-1, k, s, t}(x)-d_{j, k-1, s, t}(x)+d_{j-1, k-1, s, t}(x)\right| .
$$

Then

$$
\begin{aligned}
\alpha_{j k s t} & -\alpha_{j, k+1, s, t}-\alpha_{j+1, k, s, t}+\alpha_{j+1, k+1, s, t} \\
& =\left|d_{j k s t}(x)-d_{j-1, k, s, t}(x)-d_{j, k-1, s, t}(x)+d_{j-1, k-1, s, t}(x)\right| .
\end{aligned}
$$

Proof is easy and hence omitted.

\section{INCLUSION RELATIONS}

In this section, we prove some inclusion relations concerning of our newly defined double sequence spaces.

Theorem 4.1. $\mathcal{W}_{\sigma} \subset \mathcal{U}_{\sigma}$ and the reverse inclusion does not hold in general.

Proof. Let $x=\left(x_{j k}\right) \in \mathcal{W}_{\sigma}$. Then there exist integers $p_{0}, q_{0}$ such that

$$
\left.\begin{array}{l}
\sum_{p>p_{0}}\left|\phi_{p q s t}(x)\right|<1 \text { for each } q, \\
\sum_{q>q_{0}}\left|\phi_{p q s t}(x)\right|<1 \text { for each } p, \\
\sum_{p>p_{0}} \sum_{q>q_{0}}\left|\phi_{p q s t}(x)\right|<1 .
\end{array}\right\}
$$

Now we have to show that $\phi_{p q s t}(x)$ is bounded for every fixed $p, q$ such that $0 \leq p \leq p_{0}, 0 \leq q \leq q_{0}$. From (4.12), we have $\left|\phi_{p q s t}(x)\right|<1$ for each fixed 
$p>p_{0}, q>q_{0}$. Since

$$
\begin{aligned}
\sum_{p=0}^{\infty} \sum_{q=0}^{\infty}\left|\phi_{p q s t}(x)\right|= & \sum_{p=0}^{p_{0}} \sum_{q=0}^{q_{0}}\left|\phi_{p q s t}(x)\right|+\sum_{p=p_{0}+1}^{\infty} \sum_{q=0}^{q_{0}}\left|\phi_{p q s t}(x)\right| \\
& +\sum_{p=0}^{p_{0}} \sum_{q=q_{0}+1}^{\infty}\left|\phi_{p q s t}(x)\right|+\sum_{p>p_{0}}^{\infty} \sum_{q>q_{0}}^{\infty}\left|\phi_{p q s t}(x)\right|
\end{aligned}
$$

then in view of (4.12) to show that $x \in \mathcal{U}_{\sigma}$ it is sufficient to prove that $\sup _{s, t} \sum_{p=0}^{p_{0}} \sum_{q=0}^{q_{0}}\left|\phi_{p q s t}(x)\right|<\infty$, but this follows, since $x=\left(x_{k j}\right)$ is bounded.

Hence $\sup _{s, t} \sum_{p=0}^{\infty} \sum_{q=0}^{\infty}\left|\phi_{p q s t}(x)\right|<\infty$, i.e., $\mathcal{W}_{\sigma} \subset \mathcal{U}_{\sigma}$.

Now, we show that the reverse inclusion does not hold in general.

Let $\sigma(n)=n+1$. Define the double sequence $x=\left(x_{j k}\right)$ by

$$
x_{j k}=\left\{\begin{array}{l}
\frac{1}{j+1}\left[1+(-1)^{j}\right], 0 \leq k \leq j ; \\
0, j<k .
\end{array}\right.
$$

Then $x \notin \mathcal{W}_{\sigma}$ but it is trivial that $x \in \mathcal{U}_{\sigma}$.

This completes the proof of the theorem.

THEOREM 4.2. $\mathcal{L}_{\sigma} \subset \mathcal{W}_{\sigma}$ and the reverse inclusion does not hold in general.

Proof. The result follows from the chain of inequalities. We know that

$$
\begin{aligned}
\sum_{p=1}^{\infty} \sum_{q=1}^{\infty}\left|\phi_{p q s t}(x)\right|= & \sum_{p=1}^{\infty} \sum_{q=1}^{\infty} \frac{1}{p(p+1) q(q+1)} \mid \sum_{j=1}^{p} \sum_{k=1}^{q} j k\left[x_{\sigma^{j}(s), \sigma^{k}(t)}\right. \\
& \left.-x_{\sigma^{j-1}(s), \sigma^{k}(t)}-x_{\sigma^{j}(s), \sigma^{k-1}(t)}+x_{\sigma^{j-1}(s), \sigma^{k-1}(t)}\right] \mid \\
\leq & \sum_{j=1}^{\infty} \sum_{k=1}^{\infty} j k \mid x_{\sigma^{j}(s), \sigma^{k}(t)}-x_{\sigma^{j-1}(s), \sigma^{k}(t)}-x_{\sigma^{j}(s), \sigma^{k-1}(t)} \\
& +x_{\sigma^{j-1}(s), \sigma^{k-1}(t)} \mid \sum_{p=j}^{\infty} \sum_{q=k}^{\infty} \frac{1}{p(p+1) q(q+1)} \\
\leq & \sum_{j=1}^{\infty} \sum_{k=1}^{\infty} \mid x_{\sigma^{j}(s), \sigma^{k}(t)}-x_{\sigma^{j-1}(s), \sigma^{k}(t)}-x_{\sigma^{j}(s), \sigma^{k-1}(t)} \\
& +x_{\sigma^{j-1}(s), \sigma^{k-1}(t)} \mid .
\end{aligned}
$$

Hence the result follows.

The following example shows that the reverse inclusion need not be true. 
Let $\sigma(n)=n+2$. Consider the double sequence $x=\left(x_{j k}\right)$ defined by

$$
x_{j k}=\left\{\begin{array}{l}
1, \text { if } j \text { is odd for all } k \\
0, \text { otherwise. }
\end{array}\right.
$$

Then $\phi_{p q s t}(x)=0$ for all $p, q \geq 1$. Thus $x \in \mathcal{W}_{\sigma}$ but $x \notin \mathcal{L}_{\sigma}$.

This completes the proof of the theorem.

Theorem 4.3. $\mathcal{W}_{\sigma} \subset\left[\mathcal{V}_{\sigma}\right]$ if conditions (ii) and (iii) of Lemma 3.2 hold. Also $\left[\mathcal{V}_{\sigma}\right]-\lim x=\sigma-\lim x$ for all $x \in \mathcal{W}_{\sigma}$.

Proof. Suppose that $x=\left(x_{j k}\right) \in \mathcal{W}_{\sigma}$. Then

$$
\alpha_{g h s t}=\sum_{j=g}^{\infty} \sum_{k=h}^{\infty}\left|d_{j k s t}(x)-d_{j-1, k, s, t}(x)-d_{j, k-1, s, t}(x)+d_{j-1, k-1, s, t}(x)\right|
$$

$$
\longrightarrow 0 \text { as } g, h \longrightarrow \infty \text {, uniformly in } s, t \text {. }
$$

and

$$
d_{j k s t}(x) \longrightarrow \ell \text {, say, as } j, k \longrightarrow \infty \text { uniformly in } s, t,
$$

that is, $\sigma-\lim x=\ell$.

In order to prove that $x \in\left[\mathcal{V}_{\sigma}\right]$, it is enough to show that condition (iv) of Lemma 3.2 holds. By Lemma 3.3 and Lemma 3.4 we have

$$
\begin{aligned}
& x_{\sigma^{j}(s), \sigma^{k}(t)}+d_{j k s t}(x)-\alpha(j, k, s, t)-\beta(j, k, s, t) \\
& \quad=j k\left[d_{j k s t}(x)-d_{j-1, k, s, t}(x)-d_{j, k-1, s, t}(x)+d_{j-1, k-1, s, t}(x)\right]
\end{aligned}
$$

and

$$
\begin{gathered}
\left|d_{j k s t}(x)-d_{j-1, k, s, t}(x)-d_{j, k-1, s, t}(x)+d_{j-1, k-1, s, t}(x)\right| \\
=\alpha_{j k s t}-\alpha_{j, k+1, s, t}-\alpha_{j+1, k, s, t}+\alpha_{j+1, k+1, s, t} .
\end{gathered}
$$

So that we have

$$
\begin{aligned}
& \frac{1}{p q} \sum_{j=1}^{p} \sum_{k=1}^{q}\left|x_{\sigma^{j}(s), \sigma^{k}(t)}+d_{j k s t}(x)-\alpha(j, k, s, t)-\beta(j, k, s, t)\right| \\
& \quad=\frac{1}{p q} \sum_{j=1}^{p} \sum_{k=1}^{q} j k\left[\alpha_{j k s t}-\alpha_{j, k+1, s, t}-\alpha_{j+1, k, s, t}+\alpha_{j+1, k+1, s, t}\right]
\end{aligned}
$$

by using Lemma 3.1 for Abel's transformation, we have

$$
\begin{aligned}
& \frac{1}{p q} \sum_{j=1}^{p} \sum_{k=1}^{q}\left|x_{\sigma^{j}(s), \sigma^{k}(t)}+d_{j k s t}(x)-\alpha(j, k, s, t)-\beta(j, k, s, t)\right| \\
& =\frac{1}{p q}\left[\sum_{j=1}^{p} \sum_{k=1}^{q} \alpha_{j k s t}-p \sum_{k=1}^{q} \alpha_{p+1, k, s, t}-q \sum_{j=1}^{p} \alpha_{j, q+1, s, t}+p q \alpha_{p+1, q+1, s, t}\right], \\
& \quad \longrightarrow 0 \text { as } p, q \longrightarrow \infty, \text { uniformly in } s, t, \text { by }(4.13) .
\end{aligned}
$$


Hence by Lemma 3.2, $x \in\left[\mathcal{V}_{\sigma}\right]$.

This completes the proof of the theorem.

ACKNOWLEDGEMEnTs.

Partial results of this paper were presented by the second author as an invited talk in the "Mathematics Workshop Days" on Summability, Sequence Spaces and Applications, during May 15-16, 2008, Istanbul. The author is grateful to the Council of Scientific and Industrial Research (India) for providing the travel grant, under Grant No.TG/3021/08-HRD.

Research of the first author was supported by the Department of Science and Technology, New Delhi, under Grant No. SR/S4/MS:505/07; and the research of the second author was supported by the Department of Atomic Energy, Government of India under the NBHM-Post Doctoral Fellowship Programme Number 40/10/2008-R\&D II/892.

Authors are grateful to the referees for their valuable comments.

\section{REFERENCES}

[1] B. Altay and F. Başar, Some new spaces of double sequences, J. Math. Anal. Appl. 309 (2005), 70-90.

[2] M. Başarir, On the strong almost convergence of double sequences, Period. Math. Hungar. 30 (1995), 177-181.

[3] C. Çakan, B. Altay and Mursaleen, The $\sigma$-convergence and $\sigma$-core of double sequences, Appl. Math. Lett. 19 (2006), 1122-1128.

[4] G. Das, B. Kutter and S. Nanda, Some sequence spaces and absolute almost convergence, Trans. Amer. Math. Soc. 283 (1984), 729-739.

[5] G. Das and S. K. Sahoo, A generalization of strong and absolute almost convergence, J. Indian Math. Soc. (N.S.) 58 (1992), 65-74.

[6] G. H. Hardy, On the convergence of certain multiple series, Proc. London Math. Soc. (2) 1 (1903), 124-126.

[7] F. Móricz and B. E. Rhoades, Almost convergence of double sequences and strong regularity of summability matrices, Math. Proc. Cambridge Philos. Soc. 104 (1988), 283-294.

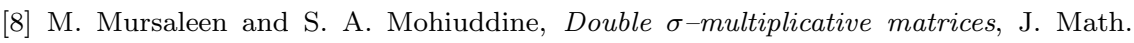
Anal. Appl. 327 (2007), 991-996.

[9] M. Mursaleen and S. A. Mohiuddine, Regularly $\sigma$-conservative and $\sigma$-coercive four dimensional matrices, Comput. Math. Appl. 56 (2008), 1580-1586.

[10] M. Mursaleen, On some new invariant matrix methods of summability, Quart. J. Math. Oxford Ser. (2) 34 (1983), 77-86.

[11] M. Mursaleen, Matrix transformations between some new sequence spaces, Houston J. Math. 9 (1983), 505-509.

[12] A. Pringsheim, Zur theorie der zweifach unendlichen Zahlenfolgen, Math. Ann. 53 (1900), 289-321. 
M. Mursaleen

Department of Mathematics

Aligarh Muslim University

Aligarh 202002

India

E-mail: mursaleenm@gmail.com

S.A. Mohiuddine

Department of Mathematics

Aligarh Muslim University

Aligarh 202002

India

E-mail: mohiuddine@gmail.com

Received: 20.2.2009.

Revised: 24.3.2009. \& 10.4.2009. 